\title{
DEVELOPMENT AND CHARACTERIZATION OF HIGH-SENSITIVITY BIOINSPIRED ARTIFICIAL HAIRCELL SENSOR
}

\author{
Nannan Chen, Jack Chen, Jonathan Engel, Saunvit Pandya, Craig Tucker, and Chang Liu \\ Micro Actuators, Sensors, and Systems Group, University of Illinois at Urbana-Champaign
}

Urbana, IL, USA

\begin{abstract}
We report the development of a high sensitivity artificial haircell (AHC) sensor that employs high aspect-ratio cilium (up to $700 \mu \mathrm{m}$ tall) made of SU-8 epoxy and silicon piezoresistive strain sensors. In this work, we demonstrate the application of the artificial haircell for underwater flow sensing. For device characterization, we have performed deflection testing, resonant frequency testing, sensitivity threshold testing and preliminary dipole field response experiments. We have demonstrated a flow rate sensitivity of $2 \mathrm{~mm} / \mathrm{s}$.
\end{abstract}

\section{INTRODUCTION}

In numerous biological species, the hair cell sensor serves as the building block to fill a variety of sensing needs, including sensing vibration, flow, and touch [1-2]. Figure 1 shows the example of a spider's flow receptor hair cell sensor. It is based on organic materials but can detect sub-nm dendritic displacement and the slight breezes caused by nearby predators or prey. The functions of hair cells have been very closely studied by biologists over the years, but only in recent years, with the development of micromachining techniques have researchers started to mimic the stimulus-transmission mechanism of biological sensing system [35]. Existing haircell sensors are based on various sensing methods, but the sensors are either not sensitive enough to detect very low flow speed (on the order of several $\mathrm{mm} / \mathrm{s}$ ) or they require parallel arrays of sensors to achieve such sensitivity. In this work, we present the design of an AHC sensor that is both highly sensitive and efficient, that is we are able to detect very low flow speed using a single sensor.

\section{DEVICE DESIGN}

The device mimics the structure of a biological hair cell sensor. It consists of a cilium-like hair sitting on a paddle-shaped cantilever with doped silicon strain gauges at the base. A schematic drawing of the $\mathrm{AHC}$ is shown in Figure 2. Figure 3 shows an SEM of an actual device.
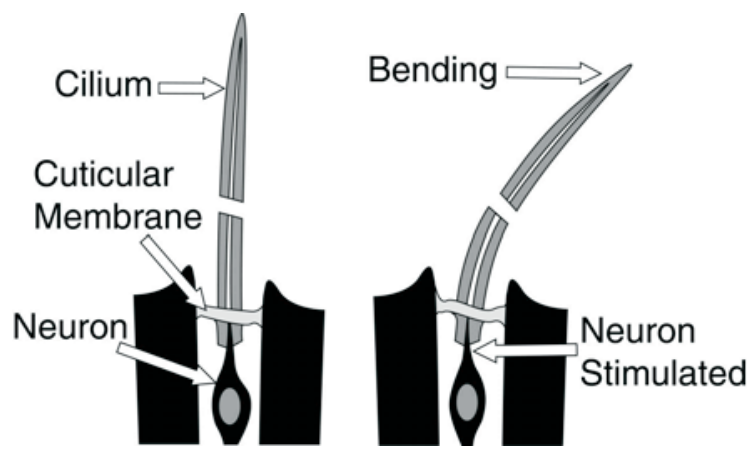

Figure 1. Schematic diagram of a haircell sensor. A cilium is attached to a neuron. When the cilium is displaced by mechanical stimulus (originating from vibration, flow impingement, contact, etc), the neuron provides electrical output.
For the cantilever-cilium configuration, the drag force acting on the cilium is completely transferred to the distal end of the cantilever. The system can then be simplified to a moment loaded cantilever:

$$
\varepsilon=\frac{6 M}{E w t^{2}}
$$

where $\varepsilon$ is the strain, $M$ is the moment, $E$ is the Young's modulus for silicon, $w$ is the cantilever width and $t$ is the cantilever thickness. The thickness is chosen to be $2 \mu \mathrm{m}$ for fabrication feasibility and good sensitivity. Based on existing signal processing circuitry and achievable piezoresistive gauge factors, we assume the minimum strain the system can detect is 2.5 microstrain. Given a desired minimum detectable flow speed of $1 \mathrm{~mm} / \mathrm{s}$, $M$ is now a function of the cilium height and diameter. Figure 4 is a 3-D map that shows what cantilever width design will satisfy these requirements as a function of cilium height and diameter.

For single spin SU-8 process, $600 \mu \mathrm{m}$ height and $80 \mu \mathrm{m}$ diameter are reasonable values for cilium dimensions. Based on these cilium dimensions, the cantilever width is determined to be $40 \mu \mathrm{m}$. The length of the cantilever is chosen so that the resonant frequency is on the order of several $\mathrm{KHz}$.

The strain gauges are achieved by ion implantation. The ion implantation is performed on very lightly doped n-type sample with boron as dopant. To optimize the performance of the stain gauge, we have chosen the ion implantation parameters so that the doping depth is approximately $1 / 3$ of the total beam thickness and the doping concentration is on the order of $1 \times 10^{20} \mathrm{~cm}^{-3}[6]$. The effective gauge factor is experimentally determined to be 33.6 , which includes all contact and parasitic resistances. The output of the sensor is read out using a Wheatstone bridge. All resistors of the Wheatstone bridge are defined on chip during ion implantation to minimize the thermal noise of the flow sensor.

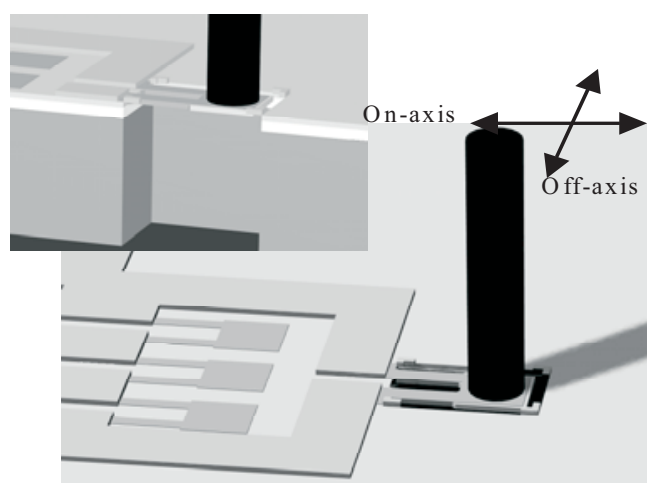

Figure 2. Perspective side view of an artificial haircell (AHC) sensor. A high aspect ratio hair is located at the distal end of a silicon paddle. 


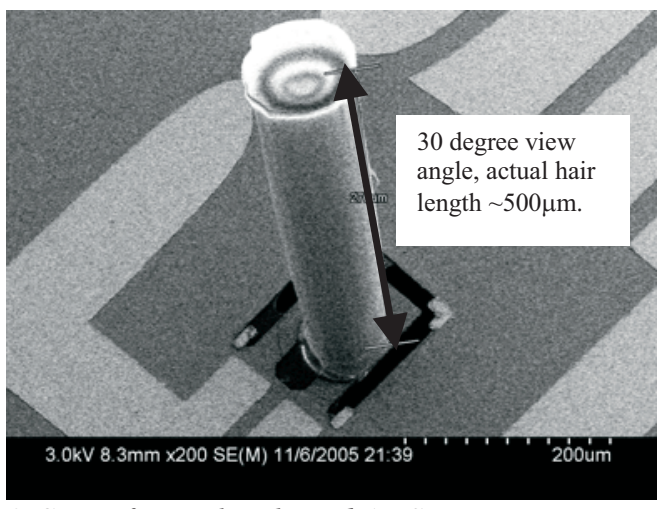

Figure 3. SEM of a single released AHC sensor.

\section{DEVICE FABRICATION}

The devices are fabricated on the SOI wafers with $2 \mu \mathrm{m}$ device thickness, $2 \mu \mathrm{m}$ oxide, and $300 \mu \mathrm{m}$ handle thickness. The cilia are made of high aspect ratio SU-8 structures with $80 \mu \mathrm{m}$ diameter and $500-700 \mu \mathrm{m}$ height. SU-8 is chosen for its ability to form rigid high aspect ratio structures using common lithography tools. The process flow is demonstrated in the Figure 5.

First, we send out the SOI wafer for ion implantation (Fig. 5(a)). After getting the wafer back, a short drive-in is performed and at the same time a thin layer of oxide is formed to serve as the insulation layer. Then we open the contact windows to the doped silicon (Fig. 5(b)). Electrical connection is formed using gold on titanium deposition and lift-off (Fig. 5(c)). The paddle-like cantilevers are then defined by front side DRIE (Fig. 5(d)). Following that the bulk backside etching is also performed using DRIE to get the cantilevers ready for release (Fig. 5(e)). A single layer of SU-8 2075 is then spun to achieve thickness of approximately $700 \mu \mathrm{m}$ (Fig. 5(f)). For pre-exposure bake, the samples are ramped up to $105^{\circ} \mathrm{C}$ at $150{ }^{\circ} \mathrm{C} / \mathrm{hr}$ ramp rate and soaked at $105^{\circ} \mathrm{C}$. After a total bake time of 13 hours the samples are then ambient cooled to room temperature. The photolithography is done using a Karl Suss contact aligner at $365 \mathrm{~nm}$. A high-wavelength pass optical filter with cutoff frequency of $300 \mathrm{~nm}$ is used during exposure to eliminate the "T-topping" effect of the SU-8 structures (Fig. 6). The exposure dose is $3000 \mathrm{~mJ} / \mathrm{cm}^{2}$. For post-exposure bake, the samples are again ramped up to $105^{\circ} \mathrm{C}$ at $150{ }^{\circ} \mathrm{C} / \mathrm{hr}$ ramp rate and soaked at $105^{\circ} \mathrm{C}$ for half an hour. The samples are then ramped down to room temperature at a controlled rate of $15^{\circ} \mathrm{C} / \mathrm{hr}$. The development is done using designated SU-8 developer with IPA as the end point indicator. After the cilia assembly, the devices are released in BHF to free the cilium-on-cantilever structures (Fig. 5(g)).

Most of the processing steps are standard, expect for the SU8 step. SU-8 is very sensitive to processing parameters, but once the processing recipe is established, the process is very repeatable and able to achieve high device yield. One of the benefits of using SU-8 is that the process minimizes manual handling of the devices during the assembly of the cilia hence improves the device yield and makes batch fabrication of the devices possible. This is contrast to previous devices that required manual wire-bonding of individual cilia. The presented devices are fabricated in pairs oriented at $90^{\circ}$ angles to each other to allow 2-axis flow measurement.

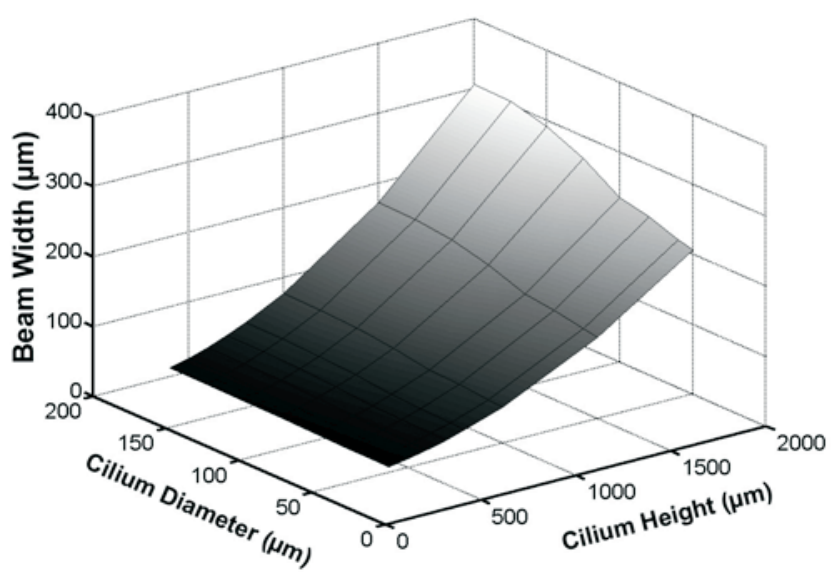

Figure 4. 3-D map shows the possible combinations of cilium height, diameter and cantilever width that satisfy our design scenario assumptions.

(a)
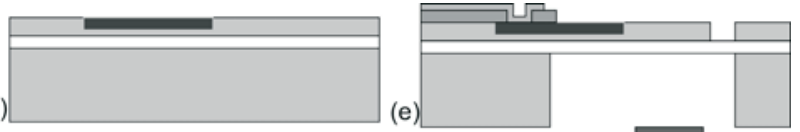

(b)

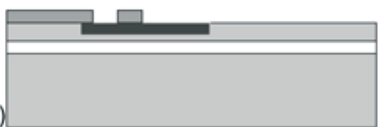

एᄄب

(c)
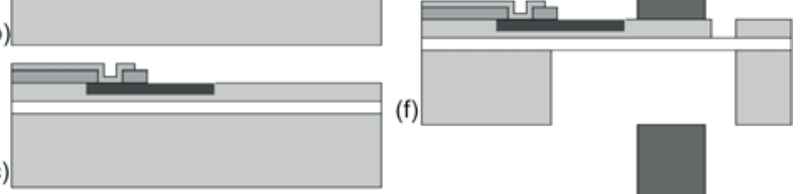

(d)
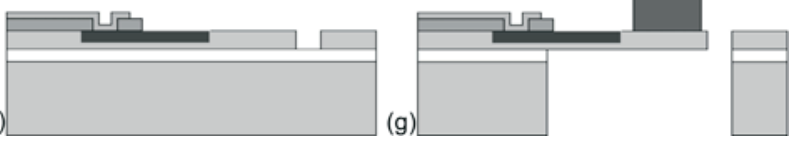

Figure 5. Fabrication process of AHC starting with a silicon-oninsulator (SOI) wafer.

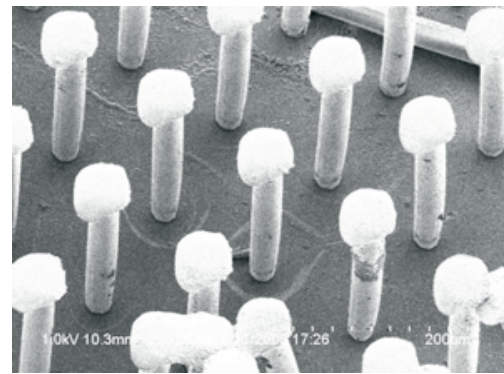

(a)

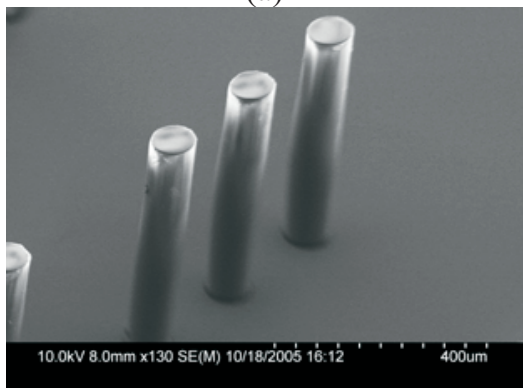

(b)

Figure 6. (a) SEM of SU-8 structures obtained without using optical filter showing the "T-Topping" effect. (b) SU-8 structures obtained using optical filter. "T-topping" effect is eliminated. 


\section{EXPERIMENT AND RESULT}

A series of experiments are done to characterize the AHC device performance and demonstrate its preliminary application as an underwater flow sensor.

\section{Tip Deflection Test}

The tip deflection test is done using the probe station. The resistance change of the strain gauge is recorded while the tip of the cilium is transversely deflected in a controlled manner. We assume that the vertical deflection of the cantilever is close to the deflection of the cilium under small angle assumption. See Figure 7 for the results of both on and off-axis deflection tests. Off-axis is defined as deflection orthogonal to the on-axis designed direction as shown in Figure 2.

Along the on-axis, the device shows very linear and sensitive response under very small defections. This is very important in our application which requires good sensitivity at very slow flow speeds. Based on the deflection response, the gauge factor is determined to be 33.6, which includes all contact and parasitic resistances. It was found that contact resistances dominated, and that an additional polyimide layer served to protect the contact areas between the metal wiring and semiconductor strain gauges during BHF release. The device has also demonstrated a very directional response with almost no response due to off-axis deflection. This is desirable in order to accurately determine flow direction as well as velocity.

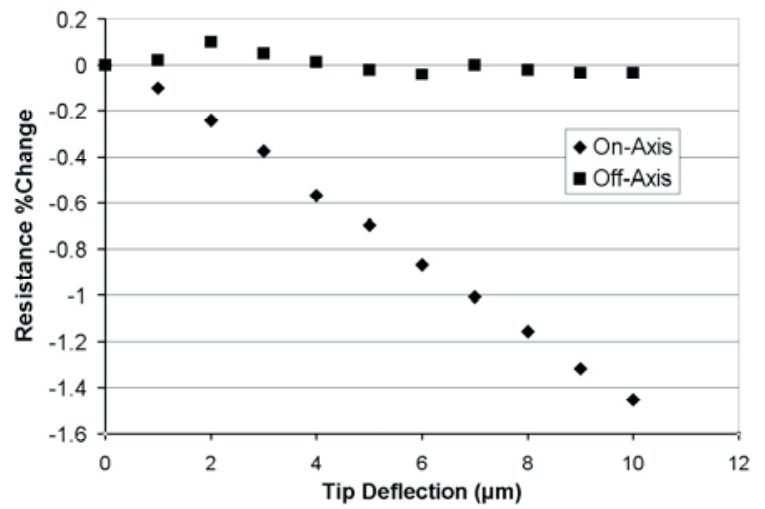

Figure 7. Tip-deflection test shows very directional response of the AHC sensor. A high rejection ratio of off-axis input is observed.

\section{Resonant Frequency Test}

The resonant frequency test is done using the AFM under Non Contact Mode. The sample is first sputtered with metal. The smooth top surface of the cilium makes it reflective to the laser beam. See Figure 8 for a typical output of the AFM. A resonant frequency of $3.07 \mathrm{KHz}$ is observed for that particular device. This agrees with our design for the resonant frequency range. This lower resonant frequency is mainly due to the cantilever structures. A resonant frequency that is orders of magnitude higher is also observed. The higher resonant frequency is mainly contributed by the rigid SU-8 cilium structure.

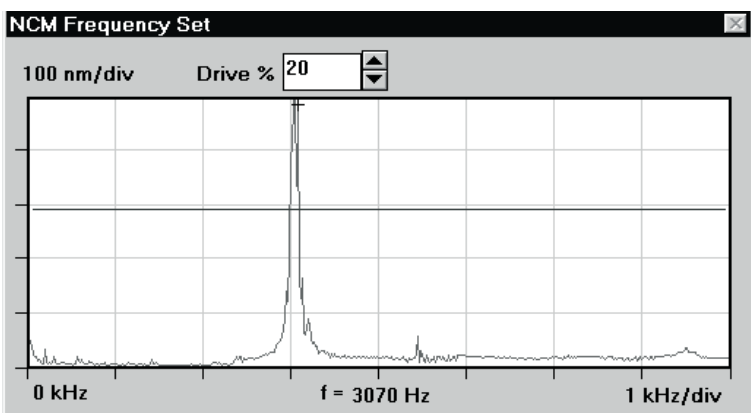

Figure 8. An AFM operated under non-contact mode is used to test the resonant frequency of AHC structure. Shown here is a typical AFM output of resonant frequency test.

\section{Sensitivity Threshold Test}

The sensitivity threshold test is done under the controlled flow condition generated by a dipole source. A dipole source is a vibrating sphere that generates mainly local, incompressible flow in the near field. Shown in Figure 9 is the sensor output vs. dipole acceleration sweep. This test is controlled by a LabVIEW program in order to quickly vary the dipole output while measuring the AHC response. The sensor output hits the noise floor around $45 \mathrm{~m} / \mathrm{s}^{2}$ of dipole acceleration. Based on the simplified near field dipole model calculation, this acceleration is translated to approximately $2 \mathrm{~mm} / \mathrm{s}$ flow speed [7]. This has demonstrated the sensor's capability of performing accurate low flow speed detection.

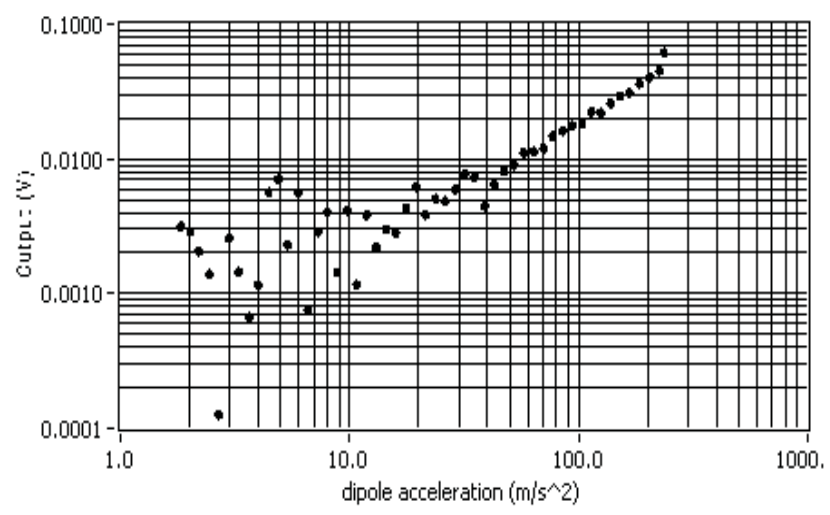

Figure 9. Sensor output vs. dipole acceleration shows the noise floor around $45 \mathrm{~m} / \mathrm{s}^{2}$, which translates to $2 \mathrm{~mm} / \mathrm{s}$ flow speed.

\section{Preliminary Dipole Localization Experiment}

The localization experiment is done with the vibrating dipole source sweeping across the position of the sensor. The dipole source is attached to a computer controlled motorized linear stage allowing accurate positioning of the dipole relative to the sensor. See Figure 10 for the sensor output vs. the position of the dipole source. A peak is observed when the dipole source is directly above the position of the sensor as expected by the dipole model. With further calibration and more sophisticated signal processing, the AHC sensors can be utilized in the precise localization and tracking of a moving object. 
Dipole Experiment $(75 \mathrm{hz})$

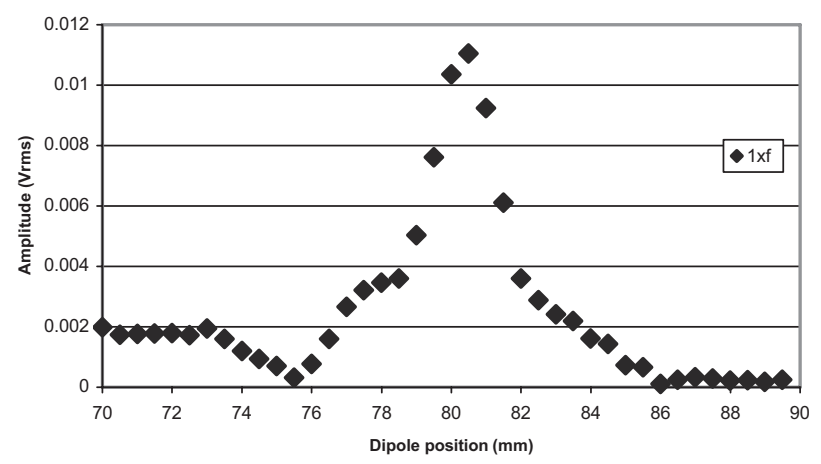

Figure 10. The sensor was used to "visualize" the velocity field created by an oscillating dipole source.

\section{DISCUSSION AND CONCLUSION}

Even though the device fabrication process is well established, there are still several challenges we are facing that can affect the output and performance of the devices.

During the photolithography of the cilia, since we are trying to focus over the range of several hundred microns, misalignment is inevitable. Usually, we are able to get the cilium close to the center of the paddle. For example in Figure 3, the cilium is partially offset on the cantilever, this has shortened the effective length of the cantilever, but since the length of the cantilever is not a determinant factor in the sensitivity of the sensor, it should not affect the sensitivity too much. However the misalignment may contribute to the asymmetric moment loading of the cantilevers, which may lead to lower sensitivity and asymmetry of the sensor response.

The high sensitivity of the sensor is achieved at the compromise of device robustness. To improve the robustness, new packaging schemes and protection features are being developed, as well as new materials for the hair structure. Our current work also involves using a pair of AHC sensors oriented orthogonal to each other to allow flow angle detection with precision as high as 1 degree (Fig. 11). Bio-inspired approaches such as covering the cilia in a gelatinous cupula are also being explored.

We have developed a high sensitivity artificial hair cell sensor that is able to detect flow speed down to the order of several $\mathrm{mm} / \mathrm{s}$. This will make the sensor a very valuable candidate in the study of the biological world and in creating bio-inspired artificial sensing organs such as an artificial lateral line.

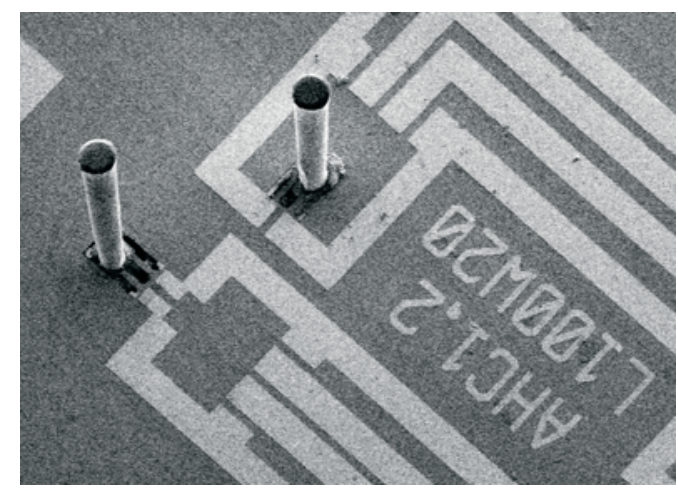

Figure 11. A pair of AHC sensors oriented orthogonally for resolving angular flow information.

\section{ACKNOWLEDGMENT}

Special thanks to DARPA BioSenSE and AFOSR Bioinspired Concept programs.

\section{REFERENCES}

[1] J. T. Albert, O. C. Friedrich, H.-E. Denchant, and F. G. Barth, "Arthropod Touch Reception: Spider Hair Sensilla as Rapid Touch Detectors," Journal of Comparative Physiology A, vol. 187, pp. 303-312, 2001.

[2] F. G. Barth, "Spider Mechanoreceptors," Current Opinion inNeurobiology, vol. 14, pp. 415-422, 2004.

[3] Y. Ozaki, T. Ohyama, T. Yasuda, and I. Shimoyama, "Air flow sensor modeled on wind receptor hairs of insects," presented at IEEE International Conference On MEMS, 2000.

[4] J. v. Baar, M. Dijkstra, R. Wiegerink, T. Lammerink, R. d. Boer, and G. Krijnen, "Arrays of cricket-inspired sensory hairs with capacitive motion detection," presented at IEEE Inernational Conference on MEMS, Miami Beach, FL, 2005.

[5] J. Engel, J. Chen, N. Chen, and C. Liu, "Development and Characterization of an Artificial Hair Cell Based on Polyurethane Elastomer and Force Sensitive Resistors," The 4th IEEE International Conference on Sensors, Irvine, California, 31 Oct- 1 Nov, 2005.

[6] J. A. Harley and T. W. Kenny, "1/f Noise Consideration for the Design and Process Optimization of Piezoresistive Cantilevers," JMEMS, vol 9, no. 2, June 2000.

[7] J. Chen, J. Engel, N. Chen, S. Pandya, S. Coombs, and C. Liu, "Artificial Lateral Line and Hydrodynamic Object Tracking," MEMS 2006 Conference, Istanbul, Turkey , January 22 - 26, 2006 